\title{
Filariasis subcutánea
}

\author{
Subcutaneous filariasis \\ Daniel Guerrero Enciso, ${ }^{*}$ Carolina González Vergara, ${ }^{*}$ Olivia Bieletto Trejo, ${ }^{\ddagger}$ Agustín Luna Tovar ${ }^{\S}$ \\ Citar como: Guerrero ED, González VC, Bieletto TO, Luna TA. Filariasis subcutánea. \\ Acta Med Grupo Angeles. 2021; 19 (2): 276-279. https://dx.doi.org/10.35366/100455
}

\section{Resumen}

Las filariasis son un conjunto de enfermedades infecciosas que afectan fundamentalmente el tejido linfático y piel, producidas por nemátodos de la familia Filariidae. Existen alrededor de 200 tipos de filarias parásitas pero sólo unas pocas parasitan al hombre transmitiéndose a través de la picadura de un insecto vector. La Organización Mundial de la Salud las ha considerado como "enfermedades desatendidas". El diagnóstico se basa en la demostración de las microfilarias en muestras de sangre o de piel en donde pueden ser visualizadas con estudios auxiliares de imagen. La mortalidad es baja pero no así las consecuencias sociosanitarias. Se presenta el caso de un hombre de 63 años a quien se realiza diagnóstico de filariasis oncocerquiana, evaluando los parásitos vivos en rastreos ultrasonográficos de alta resolución, en un nódulo cutáneo de la nuca, de cuatro años de evolución, comprobando su existencia en estudio histopatológico.

Palabras clave: Filariasis, filaria, oncocercosis, onchocerca.

\section{Abstract}

Filariasis is a group of parasitic diseases mainly affecting the lymphatic system and skin, produced by nematodes of the Filarioidea family. There are almost 200 different types of filaria worldwide and just a few parasitizing human beings. Transmission being through insects as a vector. Filariasis are considered as "neglected diseases" by the World Health Organization. Diagnosis is made by demonstrating microfilariae in circulating blood or skin where it can be visualized with auxiliary imaging methods. Although mortality is low, the morbidity associated is not only for the physically disabilities, but also mental, social, and financial losses that contribute to stigma and poverty. In this article, the case of a 63 years old man with onchocercal filariasis is presented, evaluating live parasites by scanning a cutaneous nodule in the nape with an evolution of four years histologically documented.

Keywords: Filariasis, filaria, onchocerca, onchocerciasis.

y miden entre 1 y $4 \mathrm{~cm}$ de longitud. Las filarias causan diversos cuadros clínicos, característicos de cada especie individual. ${ }^{1}$

En el plano de la taxonomía, según la clasificación de Charles Linné que data de 1735, las filarias forman parte de los nemátodos del mismo tipo que los vermes intestinales. Constituyen un problema importante de salud pública en países de alta marginación. La mortalidad asociada a las filariasis es baja, pero las consecuencias sociosanitarias de sus manifestaciones crónicas son importantes, ya que producen gran discapacidad en los sujetos que las
* Departamento de Radiología e Imagen.
₹ Jefe del Departamento de Anatomía Patológica.
§ Departamento de Cirugía General.

Hospital Ángeles Mocel. Ciudad de México. México.
Correspondencia:

Dr. Daniel Guerrero Enciso

Correo electrónico: dage9108@gmail.com

Aceptado: 22-06-2020.

www.medigraphic.com/actamedica 
padecen, lo que conlleva una baja considerable en la capacidad laboral.

Las filariasis están clasificadas como "Enfermedades desatendidas" (Neglected Tropical Diseases) por la Organización Mundial de la Salud (OMS), y se presentan en nuestro país y otros continentes, de ahí la importancia del presente caso. ${ }^{1,2}$

\section{PRESENTACIÓN DEL CASO}

Hombre de 63 años, originario y residente de la Ciudad de México, con antecedente de múltiples viajes a países de Asia, Europa, Centroamérica y Sudamérica en los últimos 10 años. No cuenta con antecedentes de enfermedades crónico-degenerativas.

Inicia su padecimiento cinco años previos al identificar incidentalmente aumento de volumen en la nuca, localizado por debajo del nivel de la apófisis espinosa de C7, duro, con bordes bien definidos y aparentemente delimitado a tejido celular subcutáneo, con un tamaño de $1 \mathrm{~cm}$. No hay alteración de la temperatura o coloración local. No se acompaña de dolor o algún otro síntoma concomitante.

La lesión no presentó cambios en su morfología durante dos años, por lo cual su primer diagnóstico fue de lipoma.

A los cuatro años se programa para resección de lipoma, con previo estudio ultrasonográfico de control, dirigido, utilizando transductor lineal de alta frecuencia $(17 \mathrm{MHz})$, documentando un nódulo subcutáneo circunscrito, de bordes definidos, con paredes delgadas midiendo $7.6 \times$ $5.2 \times 7.5 \mathrm{~mm}$. En el interior se observan ecos lineales, con constante movimiento, no vasculares en evaluación Doppler color, correspondientes a larvas vivas de filaria (Figura 1).

La lesión se reseca en su totalidad con diagnóstico histopatológico de filariasis subcutánea oncocerquiana, observando el quiste que contiene numerosos parásitos, acentuado infiltrado inflamatorio agudo y crónico perinodular (Figuras 2 y 3 ).

\section{Epidemiología}

En términos generales, los géneros de filarias de interés sanitario para el ser humano son: Wuchereria (la especie W. bancrofti); Brugia (especies B. malayi y B. timori); Loa (especie Loa loa); y Onchocerca (especie O. volvulus). Existen algunas otras especies capaces de afectar al ser humano, pero de importancia epidemiológica mucho menor. $^{3}$

En México, las principales zonas afectadas por estas parasitosis, teniendo como enfoque la oncocercosis, se localizan en Oaxaca, Norte y Sur de Chiapas y comprenden 670 localidades con un aproximado de 159 mil habitantes, siendo la mayoría candidatos a tratamiento médico, habiéndose establecido un programa de dosis semestrales de ivermectina y después cuatro dosis al año acelerando los buenos resultados para eliminar la categoría de enfermedad endémica.

Después del año 2012, y tras años dedicados a un programa de erradicación nacional con énfasis en zonas endémicas, se logró un índice de reducción absoluta de casos de $87.8 \%$, incluso llegando a tener únicamente reporte de dos casos para el siguiente año. Por lo que, a partir de 2013, la OEPA (Onchocerciasis Elimination Program for the Americas) a cargo de la OMS recomienda la suspensión de tratamiento masivo hasta la actualidad al registrar mejoría en resultados de erradicación. ${ }^{4}$

El diagnóstico definitivo se realiza por detección de los parásitos, por lo general más fácil en el caso de las microfilarias que de los parásitos adultos. Muchas de las infecciones no presentan microfilaremia en sangre (filariasis oculta), lo que obliga al diagnóstico indirecto, por la clínica, eosinofilia y detección de IgE elevada. La demostración de anticuerpos antifilaria debe valorarse de manera cuidadosa, pues presentan reacciones cruzadas con otros nemátodos y pueden existir falsos positivos. También se puede recurrir a técnicas confirmatorias más complejas, como detección de ADN por técnicas de
Figura 1:

Estudio ultrasonográfico de alta resolución que demuestra nódulo quístico con larvas de filaria en el interior, vivas (valoradas en tiempo real), circunscrito, no vascularizado.

A) Corte longitudinal.

B) Corte transverso asociado a Doppler color.
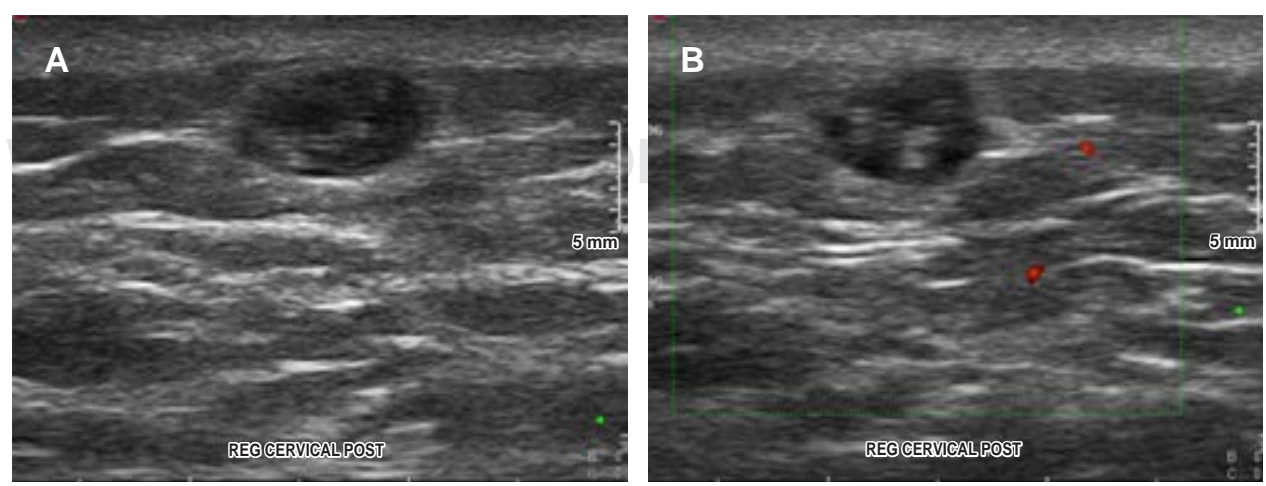
Figura 2:

A) Piel y lesión nodular subcutánea resecada, con nódulo de $7 \mathrm{~mm}$ que contiene larvas de filaria de $10 \mathrm{~mm}$ de longitud característico de nódulo oncocerquiano.

B) Montaje con tinción HE de nodulo subcutáneo profundo completamente resecado con evidencia de larvas de filaria (Nematodos).
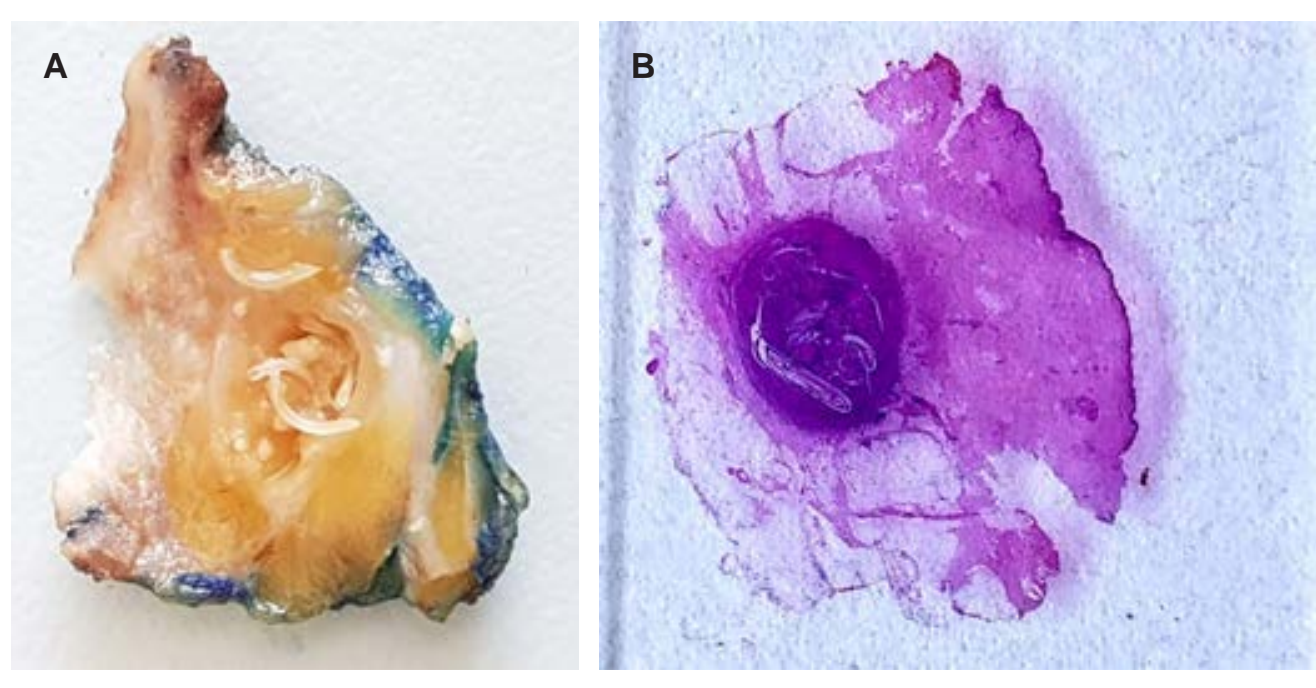
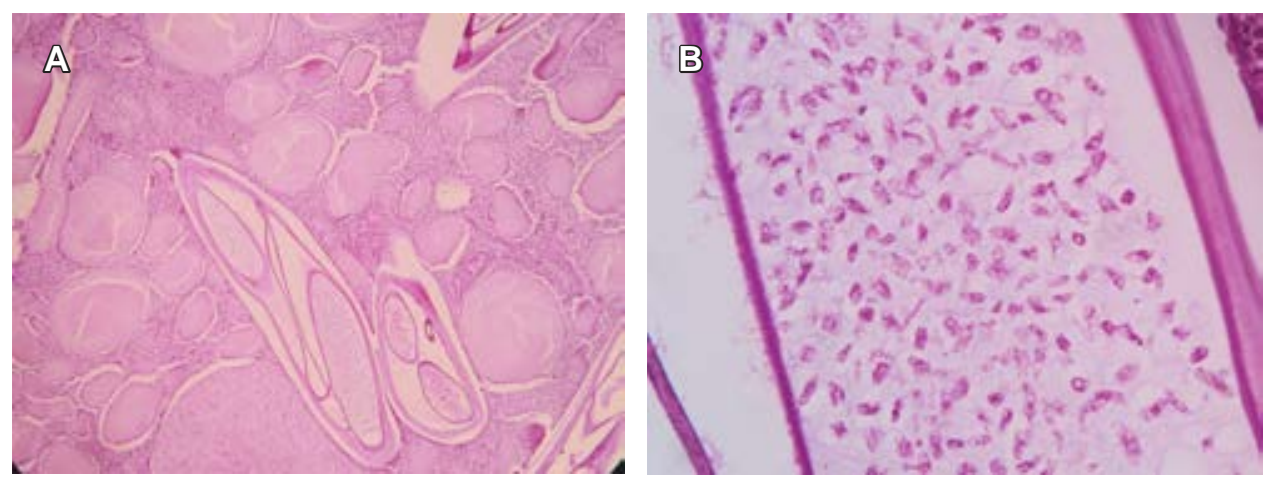

Figura 3:

A) Microfotografía de nódulo oncocerquiano observando hembras grávidas de filaria que contienen muchas microfilarias. Tinción H\&E. 5X. B) Microfotografía con fragmento del útero del parasito que contiene numerosas microfilarias (ovillos encapsulados) de siete a 10 nanómetros (milimicras) de ancho. Tinción H\&E 40X.
PCR, detección de antígenos circulantes y utilización de técnicas de imagen como ultrasonido. ${ }^{5}$

\section{DISCUSIÓN}

El mecanismo de transmisión de las filariasis son los mosquitos que pertenecen al género Anopheles.

Se estima que unos 886 millones de personas de áreas tropicales y subtropicales en 52 países están infectadas por filarias linfáticas, que clínicamente se han manifestado como "elefantiasis".

A pesar de la alta frecuencia de infección por las distintas especies de filarias en todo el mundo, son escasos los estudios realizados en la búsqueda de medicamentos eficaces y convenientes, así como regímenes de tratamiento y control eficientes.

Los ciclos biológicos tienen gran similitud entre las distintas filarias. La fase parasitaria responsable de las manifestaciones clínicas es el helminto adulto en el caso de $W$. bancrofti y B. malayi/timori, la microfilaria en el caso de O. volvulus o ambas fases en el caso de L. loa y Mansonella spp. ${ }^{6}$
Las filariasis pueden producir una amplia variedad de manifestaciones clínicas, que van desde casos aparentemente asintomáticos hasta manifestaciones más graves.

En general, la importancia clínica de esta enfermedad está dada por la elevada morbilidad a la que se asocia, sobre todo en zonas endémicas, donde producen gran discapacidad y deformidad, lo que conlleva un impacto socioeconómico muy importante.

La filariasis debe sospecharse en cualquier paciente que provenga de áreas endémicas y presente alguna de estas manifestaciones:

1. Manifestaciones cutáneas: linfangitis, edema, prurito, presbidermia.

2. Manifestaciones oculares: epífora, conjuntivitis, queratitis, pérdida de visión.

3. Afectación genitourinaria: adenopatías inguinales, hidrocele, orquitis, epididimitis, quiluria.

4. Afectación respiratoria: asma, disnea.

5. Afectación del sistema nervioso central: meningitis eosinofílica. 
6. Afectación cardiaca: miocarditis, pericarditis.

7. Afectación articular: artralgias.

Dentro de las principales filariasis en nuestro país se encuentra la oncocercosis, la cuál es consecuencia de una infección por Onchocerca volvulus. Esta filaria mide de 30 a $50 \mathrm{~cm}$ y vive bajo la piel, donde se puede agrupar para formar quistes (nódulos oncocerquianos). Esta enfermedad fue conocida como la "ceguera de los ríos", produciendo problemas de visión, ocasionando ceguera debido a que las larvas que forman los ovillos al morir lesionaban la retina. La transmisión se efectúa por la picadura de una pequeña mosca (simúlido) de la especie Simulium damnosum, que se multiplica en las cercanías de aguas de curso rápido, lo que dio nombre a la enfermedad..$^{2,7}$

El diagnóstico se basa en evidenciar las microfilarias en las muestras de piel y sangre. Para el tratamiento es necesaria la resección de los nódulos subcutáneos que constituyen la fuente de parásitos adultos. La prevención es difícil porque las pulverizaciones de productos larvicidas sobre los cursos de agua rápida deben ser repetidas de manera constante, lo que resulta muy costoso; por tal motivo, la Organización Mundial de la Salud (OMS) ha propuesto el tratamiento masivo con una dosis única de ivermectina anual a todos los habitantes de las poblacionales ubicados en las regiones afectadas. ${ }^{8}$

\section{REFERENCIAS}

1. Filariasis epidemiología y situación mundial @www.amse.es [Internet]. Disponible en: https://www.amse.es/informacionepidemiologica/112-filariasis-epidemiologia-y-situacion-mundial.

2. Díaz-Menéndez M, Norman F, Monge-Maillo B, Pérez-Molina JA. Las filariasis en la práctica clínica. Enferm Infecc Microbiol Clin. 2011; 29 (Supl. 5): 27-37.

3. Albert DC. Tratamiento de las filariosis [Trabajo de Grado]. Universidad Complutense, Facultad de Farmacia; 2018.

4. Sectorial P, Salud D. Programa de acción específico eliminación de la oncocercosis 2013-2018. 2013; Disponible en: http://www.cenaprece.salud.gob.mx/descargas/pdf/PAE EliminacionOncocercosis2013 2018.pdf.

5. Aparicio F, Vega Y, Hidalgo M, García D, De la Paz N, Martínez F. Filariasis linfática por Brugia. Presentación de un caso. Mediciego [Internet]. 2016; 22 (1): 59-54. Disponible en: http://www. revmediciego.sld.cu.

6. Lymphatic-filariasis@www.who.int [Internet].Available from: https:// www.who.int/es/news-room/fact-sheets/detail/lymphatic-filariasis.

7. López J, Valiente-Echeverría F, Carrasco M, Mercado R, Abarca K. Identificación morfológica y molecular de filarías en una comuna semi-rural de la Región Metropolitana, Chile. Rev Chilena Infectol. 2012; 29 (3): 248-289.

8. Lloveras SC. Viajeros a América del Sur. Rev Chilena Infectol. 2011; 28 (6): 520-528. 